\title{
Sprachwissenschaft
}

Izabela Bawej

Kazimierz-Wielki-Universität, Bydgoszcz

DOI: $10.19195 / 0435-5865.141 .10$

\section{Ist die Unterscheidung von Sprache und Zunge im Deutschen berechtigt? Einige Gedanken zu kulturgeprägten lexikalischen Unterschieden in der Interpretation der außersprachlichen Wirklichkeit}

\author{
Welt begegnet Menschen nicht so wie sie ist, \\ sondern so, wie sie eine bestimmte Sprach- \\ und Kulturgemeinschaft im Laufe ihrer \\ geschichtlichen Entwicklung erfahren und \\ strukturiert hat.
}

(Merten 1995: 287 zit. nach Brunzel 2002: 40)

Den Ausgangpunkt der Ausführungen bildet der unten angeführte lexikalische Fehler eines meiner Studenten der Angewandten Linguistik an der Kazimierz-Wielki-Universität in Bydgoszcz (Polnisch als Muttersprache, Deutsch als 1. Fremdsprache):

*Die Menschen sprechen verschiedene Zungen. $\rightarrow$ Die Menschen sprechen verschiedene Sprachen. (Ludzie mówią różnymi językami.)

Ich will in meinen Erörterungen darauf eingehen, dass es erhebliche Unterschiede zwischen Sprachen gibt und dass jede Sprachgemeinschaft bestimmte Bereiche von zentraler Bedeutung hat, in denen der Wortschatz besonders differenziert ist. Obwohl diese Gegebenheiten bekannt sind, möchte ich ihnen etwas Aufmerksamkeit schenken. Im Folgenden zeige ich beispielhaft auf, wie sich zwei Sprachen in semantischer Hinsicht auf kulturabhängige Art und Weise unterscheiden können und erkläre, warum im Satz des Studenten das Wort Zunge nicht passt. 
Wilhelm von Humboldt entwickelte im 19. Jahrhundert die Theorie, nach der jede Sprache die Welt in einer ganz ihr eigenen, einmaligen und unverwechselbaren Weise widerspiegelt und somit die Weltansicht (das Weltbild) des Sprachbenutzers prägt und Einfluss auf das Denken und die Kultur ihrer Sprecher nimmt. An dieser Stelle ist eine begriffliche Klärung nötig: Der erwähnte Begriff der Weltansicht (sprachlichen Weltansicht) bzw. des Weltbildes (sprachlichen Weltbildes) wird nicht als ideologische Weltanschauung verstanden. Unter einer Weltansicht (einem Weltbild) verstehen wir die außersprachliche Wirklichkeit der jeweiligen Kultur (vgl. Gipper 1993: 131; Anusiewicz 1999: 261).

Wir erfassen die Welt, ihre Objekte und Besonderheiten mit Hilfe der Sprache und wir nehmen sie mit einer bestimmten Sprache in einer bestimmten Weise wahr. Wir finden bei Humboldt die Auffassung, dass die Sprache jeder Sprachgemeinschaft eine „subjektiv-nationale Weltansicht“ widerspiegelt, indem sie die Summe aller Eindrücke und Empfindungen ihrer Mitglieder umfasst, was folgende Worte von Humboldt bestätigen: ,Jede [Sprache - I.B.] in jedem ihrer Zustände bildet das Ganze einer Weltansicht, indem sie Ausdruck für alle Vorstellungen enthält, welche die Nation sich von der Welt macht, und für alle Empfindungen, welche die Welt in ihr hervorbringt.“ (Humboldt 1968, GS 5: 433).

Nach Wilhelm von Humboldt kann man auch sagen: Wir entdecken die Welt durch die Sprache. Deswegen unterscheiden sich die Weltbilder je nach Sprache und Kultur. Alle Sprachen zielen auf die Erfassung der Welt, in der wir alle leben, sie können es nicht anders tun als auf ihre standortgebundene, historisch bedingte Weise:

Der Mensch denkt, fühlt und lebt allein in der Sprache, und muss erst durch sie gebildet werden [...] Aber er empfindet und weiss, dass sie ihm nur Mittel ist, dass es ein unsichtbares Gebiet ausser ihr gibt, in dem er nur durch sie einheimisch zu werden trachtet. [...] Alles höhere Sprechen ist ein Ringen mit dem Gedanken, in dem bald mehr die Kraft, bald die Sehnsucht fühlbar wird.

Daraus entstehen zwei höchst merkwürdige Unterschiede unter den Sprachen; der eine aus dem Grade des Gefühls jener Unzulänglichkeit, und dem Streben sie aufzuheben, der andere aus der Verschiedenheit der vorherrschenden Ansichten in der Bezeichnungsart, da die Vielseitigkeit der Gegenstände, verbunden mit der Mannigfaltigkeit der Auffassungsorgane, eine unbestimmbare Anzahl derselben erlaubt.

Einige Nationen begnügen sich gleichsam mehr an dem Gemälde, das ihre Sprachen ihnen von der Welt entwirft, und suchen nur in sie mehr Licht, Zusammenhang und Ebenmass zu bringen. Andre graben sich gleichsam mühseliger in den Gedanken ein, glauben nie genug in den Ausdruck legen zu können, ihn anpassend zu machen, und vernachlässigen darüber das in sich Vollendete der Form. Die Sprachen beider tragen dann das Gepräge davon an sich. [...]

Die andre, aus der Bezeichnungsart entstehende Gattung der Sprachunterschiede beruht auf der Ansicht der Gegenstände, und der nach ihnen gebildeten Begriffe. Ungeachtet der unendlichen Verschiedenheit derselben, liegt in allen, indem sie von einer Nation aufgefasst werden, etwas Gemeinsames der Erscheinung, das sich dem Worte, als Zeichen mittheilt. Man kann dies wohl, in groben Umrissen, so charakterisieren, dass die Wörter einer Sprache mehr sinnliche Anschaulichkeit, einer andren mehr innre Geistigkeit, einer dritten mehr nüchterne Begriffsdarlegung u.s.f. besitzen [...]. Keine jener angeführten Eigenschaften steht so vereinzelt da, und wo sie sich auch in verschiedenen Nationen gemeinsam finden, sind sie in keiner dieselben. [...]. (Humboldt 1968, GS 4: 432f.) 
Es muss noch hinzugefügt werden, dass Sprache mehr als ein Verständigungsmittel ist. Sie ist eine inhaltlich-phonetische Einheit von Geist und Materie. Sie ist Mittlerin zwischen Mensch und Welt aufgrund der Tatsache, dass erworbene Erfahrungen in Sprache umgesetzt werden. Durch Sprache ordnet und gliedert eine Sprachgemeinschaft die sie umgebende Welt. Als Spiegel der Kultur vermittelt sie „subjektive Deutungen“ einzelner Individuen und Einstellungen ganzer Sprachgemeinschaften (vgl. Dederichs 1997: 14f.; vgl. auch Anusiewicz 1999: 263; Tabakowska 2002: 27).

Generell kann man sagen, dass jede Sprache eine Weltansicht eigener Art enthält. Weltansicht der Sprache bedeutet, dass jede Sprache die Welt auf eigene Art in die Gedanken überführt, dass sie die Welt in eigenen Kategorien- und Begriffsnetzen einfängt, dass Angehörige verschiedener Kulturen unterschiedliche Vorstellungen über die Welt haben. Wer in eine Sprache hineinwächst, übernimmt unbewusst diese Sichtweisen, er sieht die Welt gleichsam durch die Brille seiner Muttersprache in bestimmter Färbung und Tönung, in spezifischer Vergrößerung oder Verkleinerung (vgl. Gipper 1992: 28; Grzegorczykowa 1999: 43).

Ganz in diesem Sinne sagt Trier (1972: 79), dass Gliederung in Bezug auf die Inhalte eine wesentliche Eigenschaft der Sprache ist: „Gliederung ist das allgemeinste und tiefste Wesensmerkmal aller Sprache. Jede Sprache gliedert das Sein auf ihre Weise, schafft damit ihr besonderes Seinsbild, setzt damit ihre, dieser einen Sprache eigentümlichen, Inhalte.“

Eine wichtige Folgerung aus dem Gesagten ist, dass die außersprachliche Wirklichkeit aus einer großen Anzahl von Wirklichkeitssegmenten besteht, die einer endlichen Anzahl von Wirklichkeitskategorien fest zugeordnet sind. Der Sprache fällt dabei die Funktion zu, den einzelnen Kategorien eine lautliche oder graphische Form zuzuordnen, die von Sprache zu Sprache anders ausfallen kann (vgl. Diller, Kornelis 1978: 27).

An dieser Stelle sollte die Frage nach der wechselseitigen Beeinflussung von Sprache und Kultur erörtert werden. Sprache ist einerseits Teil der Kultur, denn mit ihrer Hilfe benennen Menschen ständig die Welt und legen sie aus, es entsteht Lebenswirklichkeit. Insofern beeinflusst die Sprache die Kultur. Andererseits ist Sprache selbst kulturgeprägt, indem sie als Instrument zur Erfassung und Tradierung der Lebenswirklichkeit der jeweiligen Sprachgemeinschaft fungiert (vgl. Brunzel 2002: 18).

In diesem Zusammenhang verstehen wir unter Kultur die Summe der geistigen und materiellen Errungenschaften eines Volkes in einer Zeit, bezogen auf die Bereiche Musik, Kunst, Literatur, Religion, Film, Theater, Wissenschaft, Technik, Güterherstellung, Ess- und Trinkgewohnheiten, Kochkunst, Zeremonien, Landschaftsformen, soziale Organisationsformen, soziale Beziehungen usw., die auch Moral, Glaube, Sitten, bestimmte Werte, Normen, Annahmen, Einstellungen, Konventionen und die Gesamtheit aller Gewohnheiten und Verhaltensformen einschließt, welche der Mensch im Prozess der Sozialisation erwirbt und welche für eine gesamte Gesellschaft oder gesellschaftliche Gruppe Gültigkeit haben und 
an denen sich das Verhalten der Menschen ausrichtet. Kultur ist nicht etwas, was von Natur aus gegeben ist, sondern das, was durch das Leben in einer sprachkulturellen Gemeinschaft erworben wird. In diesem Sinn des Wortes Kultur hat jede Gesellschaft ihre eigene Kultur. Jede sprachliche Gesellschaft bringt im Verlauf ihrer Geschichte ihre besonderen kulturellen Gestaltungen hervor. Jede Kultur hat sich in die Richtung entwickelt, die ihr von ihren jeweiligen Umweltbedingungen vorgegeben wurde (vgl. Bawej 2014: 82f.).

Mit anderen Worten: Kultur als solche ist ein soziales Konstrukt, das alle Bereiche des gesellschaftlichen Lebens betrifft. Kultur ist ein für eine Gesellschaft oder Gruppe typisches Orientierungssystem, das aus spezifischen Symbolen gebildet wird und das Wahrnehmung, Denken und Handeln aller ihrer Mitglieder beeinflusst. Man kann also annehmen, dass Kultur die universale Fähigkeit des Menschen bezeichnet, die Welt zu deuten. Menschen deuten die Welt auf unterschiedliche Art und Weise, die auf ihre Gruppenzugehörigkeit in einem räumlich und zeitlich bestimmten System zurückzuführen ist (vgl. Brunzel 2002: 14; vgl. auch Wierzbicka 2007: 16). Die Welt an sich ist ein Strom von unterschiedlichen Reizen, der je nach Sprachgemeinschaft verschiedenartig aufgeteilt wird. Durch unsere jeweilige Kultur geben wir der Welt eine Ordnung, mit der wir die Welt sehen und strukturieren. Jede Kultur lebt in ihrer eigenen Wirklichkeit und daher ist die Erkenntnis der außersprachlichen Wirklichkeit kulturell determiniert (vgl. Lyons 1983: 268; Grzegorczykowa 1999: 43).

Aus dem oben Gesagten geht hervor, dass die Sprache einer bestimmten Gesellschaft ein wesentlicher Teil ihrer Kultur ist. In den lexikalischen Unterscheidungen, die jede Sprache vornimmt, spiegeln sich die kulturell bedeutungsvollen Merkmale von Objekten, Gegenständen, Einrichtungen und Vorgängen in der Gesellschaft, in der diese Sprache wirkt (vgl. Lyons 1989: 442). In der Natur gibt es vorgegebene Ordnungen verschiedenster Art. Das gilt für die ganze biologische und physikalische Welt. Der Mensch sieht die real existierenden Ordnungen in spezifisch menschlicher Weise und er versucht mit Hilfe seiner Sprache außersprachlich Vorgegebenes zu benennen. Deshalb werden in jeder Sprache wichtig erscheinende Merkmale hervorgehoben, andere vernachlässigt. Was aber wichtig ist oder nicht, kann in einzelnen Sprachgemeinschaften und Kulturen sehr unterschiedlich sein. Deswegen kommt es zu den spracheigentümlichen Unterteilungen und Transformationen der umgebenden Wirklichkeit, mit dem Ziel den jeweiligen Lebensbedingungen gerecht werden zu können (vgl. Gipper 1993: 10; Wierzbicka 2007: 16).

Unumstritten ist die Tatsache, dass die Sprache unsere Gedanken lenken und auf bestimmte Aspekte fokussieren kann. Jede Sprachgemeinschaft klassifiziert die Welt nach bestimmten geltenden Prinzipien: Gruppierungen können zum Beispiel nach Ähnlichkeiten vorgenommen werden (Substanz, Gestalt, Farbe etc.) oder die Sprache orientiert sich am Prinzip der Ökonomie. Es werden nur so viele Wörter wie nötig gebildet. Daher kommen in bestimmten Lebensbereichen je nach Bedarf feinere Unterteilungen vor als in anderen (vgl. Schwarz, Chur 1993: 65). 
Benutzer jeder Sprache zeigen ihr jeweils besonderes Interesse für bestimmte Dinge der Welt in entsprechenden Spezialisierungen innerhalb ihrer Sprache. Die Unterteilungen sprachlicher Art schwanken in den einzelnen Sprachen. Das gilt für alle Elemente der außersprachlichen Wirklichkeit, die in einem Land eine größere Bedeutung haben mögen als anderswo.

Der Mensch benennt mit Hilfe der Sprache nur das, was für seine jeweilige Sprachgemeinschaft zur Erfassung ihrer Umwelt relevant ist. Dies bedeutet, dass in einer Sprache eine ganz bestimmte Einstellung zur Welt zum Ausdruck gelangt. Diese Einstellung zur Umwelt, die in einer Sprache niedergelegt ist, prägt ihrerseits das Bewusstsein der Sprecher dieser Sprache (vgl. Spillmann 2000: 43).

Aus den vorangegangenen Überlegungen ergibt sich, dass manche Sprachen Unterschiede in der außersprachlichen Wirklichkeit durch verschiedene Wörter lexikalisieren und andere Sprachen solche Unterschiede sprachlich nicht beachten. Die eine Sprache unifiziert die Wirklichkeit mit Hilfe einer lexikalischen Einheit und eine andere Sprache mit Hilfe von ein paar Lexemen, was untenstehende Beispiele belegen können: Polnisch żótw, Deutsch Schildkröte, Englisch tortoise, turtle; Englisch boil, Polnisch gotować się, zagotować się, wrzeć, gotować, kipieć, zagotować, Deutsch kochen; Polnisch zazdrosny, Englisch envious, jealous, Deutsch eifersüchtig, neidisch.

Weitere Beispiele: Das Englische hat die zwei Lexeme tree und wood, wobei wood: 1. eine Ansammlung von Bäumen, 2. das Material, das aus Bäumen gewonnen wird, bedeutet (vgl. Löbner 2003: 231). Dagegen haben das Deutsche und das Polnische drei Lexeme: Deutsch Baum, Holz, Wald; Polnisch: drzewo, drewno, las. Im Polnischen existiert das Wort mgła, wobei im Deutschen je nach Dichte der Luftfeuchtigkeit sprachlich zwischen Dunst und Nebel, im Englischen zwischen haze, mist und fog begrifflich (sprachlich) differenziert wird, woraus sich sprachliche Einheiten mit unterschiedlichem Bedeutungsumfang ergeben.

In einigen Bereichen ist das semantische Raster einer Sprache anders als das der anderen. Nehmen wir nun folgendes Beispiel: Deutsch Fleisch - Englisch flesh, meat. Für den einen deutschen Inhalt Fleisch hat das Englische zwei: flesh (lebendes Fleisch) und meat (totes Fleisch). Die Anzahl der sprachlichen Zeichen ist in gewissen Bereichen unterschiedlich, so dass man zum Beispiel beim Übersetzten nicht direkt die englischen Zeichen durch deutsche ersetzen kann. Zweifellos kann ein Deutscher genau wie ein Engländer zwischen lebendem und totem Fleisch unterscheiden, aber eine der beiden Sprachgemeinschaften hat nur ein Wort dafür und die zweite hat zwei Äquivalente (vgl. Löbner 2003: 231).

Die Sprachen spiegeln zum Beispiel soziale Strukturen und kulturelle Maßstäbe wider. Einen kulturell interessanten sprachlichen Befund liefert das System der Verwandtschaftsbezeichnungen in vielen Sprachen. Die Gliederung der Verwandtschaftsbezeichnungen in verschiedenen Kulturen weist auf die Organisation der Gesellschaft hin und legt den Schwerpunkt auf bestimmte menschliche Verhältnisse und Beziehungen. 
Ein Beispiel für eine detaillierte Einteilung der Gesellschaft findet man im Hebräischen: Es gibt die Bezeichnung machatanim, was im Englischen in-laws oder angeheiratete Verwandte bezeichnet. Eine männliche (mechutan) und eine weibliche (mechatainista) Form sind vorhanden, die „männliche“ bzw. „weibliche" angeheiratete Verwandte bezeichnen. Ein differenziertes Wortfeld für die Unterscheidung zwischen Tanten und Onkeln der mütterlichen oder väterlichen Seite mit gleichzeitigem Altersbezug ist im Chinesischen zu finden: Onkel - 1. [boab'] (älterer Bruder des Vaters), 2. [Ŝdüu] (jüngerer Bruder des Vaters), 3. [iüu] (Bruder der Mutter); Tante - 1. [gügu] (Schwester des Vaters), 2. [ji] (Schwester der Mutter). Hier beinhalten die lexikalischen Unterschiede nicht nur den Aspekt der mütterlichen bzw. väterlichen Seite, sondern auch das Alter der Brüder des Vaters. Die Unterscheidung des Alters der Brüder (wegen des patriarchalischen Stammbaumes) spielt in der chinesischen Kultur eine bedeutende Rolle, was lexikalisch markiert wird (vgl. Weiss 1996: 40ff.). Zum Beispiel unterscheidet das Japanische verbal zwischen älteren und jüngeren Geschwistern. Die japanische Gesellschaft ist hierarchisch strukturiert, deswegen haben keine zwei Personen, die sozial miteinander zu tun haben, die gleiche Stellung: Ältere haben einen höheren Rang als Jüngere, Männer rangieren höher als Frauen, ältere Geschwister stehen höher als ihre jüngeren Geschwister. Solche Rangfolgen sind tief im Sozialverhalten der Japaner und in ihrer Sprache verwurzelt. Beispielsweise sind die Anredeformen unter japanischen Geschwistern mit einer Art Titel verbunden, während sich europäische Geschwister gegenseitig mit dem Vornamen anreden. In Japan werden ältere Geschwister mit einer förmlichen Bezeichnung und dem Anhängsel -san im Sinne von „Herr/Frau“ angesprochen: onêsan „Frau ältere Schwester“ bzw. onîsan „Herr älterer Bruder“. Jüngere Geschwister werden dagegen formlos mit ihren Vornamen angesprochen (vgl. Löbner 2003: 242f.).

In der einen Sprache kommen wie gezeigt Bedeutungsabgrenzungen vor, die es in einer anderen nicht gibt. Erklären wir das noch an folgendem Beispiel:

fr. poil, it. pelo, sp. pelo (Körperhaar) - fr. cheveu, it. capello, sp. cabello (Kopfhaar), dt. Haar, poln. wtos.

Den Inhaltsbereich von dt. Haar teilen sich fr. poil - cheveu, it. pelo - capello, sp. pelo - cabello. Im Polnischen gibt es wie im Deutschen ein Wort für ein beide Konzepte einschließendes Konzept. Wir können sicher sein, dass Deutsche und Polen kein anderes Sachwissen als Spanier, Italiener und Franzosen haben. Auch im Deutschen kann man ja den konzeptuellen Unterschied versprachlichen (Kopfhaar-Körperhaar). Im Unterschied zum Deutschen (und Polnischen) muss der konzeptuelle Unterschied in der Kategorisierung der außersprachlichen Wirklichkeit im Französischen, Italienischen und Spanischen versprachlicht werden (vgl. Blank 2001: 131). Der Begriffsbezirk wird in diesen Sprachen durch zwei Formative gefasst. Hier muss bemerkt werden, dass die Bedeutung eine sprachli- 
che Kategorie ist, weil sie das Abbild des Wirklichkeitsausschnittes ist, das bei den meisten Angehörigen einer Sprachgemeinschaft durch ein bestimmtes Formativ erzeugt wird, das mit der Bedeutung zur Einheit des Zeichens verbunden ist. Der Umfang der Bedeutung, d.h. auf welche Arten, auf wie viele Arten von Klassen der objektiven Realität sich ein Zeichen beziehen kann, kann von Sprache zu Sprache variieren.

Die aufgezählten Beispiele zeigen zahlreiche soziokulturelle und kulturgeschichtliche Unterschiede zwischen den Sprachen. Lexikalische Einheiten sind Spiegel der einzigartigen kulturellen Erfahrungen einer Sprachgemeinschaft. Geographische und klimatische Lage, Lebensbedingungen und Lebenskultur, Flora und Fauna etc. rufen unterschiedliche Assoziationen hervor und daher zeigt ihre Versprachlichung viele Unterschiede in den einzelnen Sprachen. Jede Sprachgemeinschaft besitzt eine ihr eigene Realienspezifik, die ihren Niederschlag in der Lexik findet.

Um eine unterschiedliche Konzeptualisierung und daraus resultierende Versprachlichung handelt es sich auch in dem oben angeführten Beispiel:

\section{Ludzie mówia różnymi językami.}

*Die Menschen sprechen verschiedene Zungen.

$\rightarrow$ Die Menschen sprechen verschiedene Sprachen.

Versuchen wir nun zu erklären, warum und in welchen Kontexten die Wörter Zunge und Sprache vorkommen.

Die meisten Lernenden neigen zur wörtlichen Übersetzung. indem sie ihre muttersprachig formulierten Gedanken Wort für Wort wiedergeben. Wenn wir Wörter oder Wortgruppen aus einer Sprache in die andere übersetzen, müssen wir nach äquivalenten zielsprachlichen Einheiten suchen, d.h. die Bedeutung der Lexeme eindeutig feststellen. Da viele Wörter polysemen Charakter haben, d.h. sie verfügen über mehrere Sememe, ist es in manchen Fällen für den Lernenden schwierig, sich für eines von zwei oder mehreren Äquivalenten in der Zielsprache zu entscheiden, die in einem Wörterbuch stehen. Die Bedeutung eines solchen Wortes ergibt sich erst aus dem Zusammenhang des Textes (aus dem sprachlichen Kontext). Wenn dem Lernenden verschiedene Übersetzungsäquivalente zur Verfügung stehen, kann die Mehrdeutigkeit (Vieldeutigkeit) nur durch Beachtung des Kontexts aufgelöst werden. Angenommen, der Lernende will den Satz Ludzie mówia różnymi językami übersetzen und schlägt unter dem Stichwort język zum Beispiel im Langenscheidts Taschenwörterbuch (1994: 134) nach, so werden hier als Äquivalente Zunge und Sprache angegeben (Polnisch język - Deutsch Zunge, Sprache). In dem gegebenen Beispiel gibt es für ein polnisches Wort verschiedene, hier zwei, Übersetzungsäquivalente im Deutschen. Der Lernende glaubt oft, dass es sich bei den Wörterbuchangaben um synonyme Wörter handelt. An dieser Stelle muss man betonen, dass zwei, drei oder mehr Wörter in einer Sprache, die im Wörterbuch unter einem bestimmten 
Stichwort stehen, keine Synonyme sind und nicht den gleichen semantischen Bereich umfassen.

Bei der Übersetzung aus dem Polnischen ins Deutsche muss sich der Lernende zwischen den einzelnen Lexemen der Zielsprache entscheiden. Dabei ist zumindest der gesamte Satz zu berücksichtigen. Der Lernende übersetzt aber den Satz Glied für Glied und wählt das erste Äquivalent: *Die Menschen sprechen verschiedene Zunge.

Wobei Zunge im Deutschen in diesem Kontext das bewegliche Muskelorgan in der Mundhöhle ist. Korrekt ist: Die Menschen sprechen verschiedene Sprachen, weil Sprache hier ein System von Lauten, Wörtern und Regeln für die Bildung von Sätzen bedeutet, das man benutzt, um sich mit anderen zu verständigen.

Der deutschsprechende Pole muss zwischen den zwei deutschen Äquivalenten des polnischen język die richtige Wahl treffen, wenn er einen semantisch akzeptablen Satz bilden will:

- Polnisch: język - 1. elastyczny narząd w jamie ustnej, sktadający się z mięśni, pokryty błona śluzowa, organ smaku, jeden z najważniejszych narządów mowy u lu$d z i[\ldots]$; 2. zasób wyrazów, zwrotów i form określanych przez reguly gramatyczne, używanych w celu porozumiewania się a) przez członków jednego narodu, społeczeństwa [...], b) przez ludzi pewnego środowiska, zawodu regionu, [...], 3. przedmiot przypominajacy ksztaltem język [...] (Nowy słownik języka polskiego 2002: 294).

- Deutsch: Sprache - 1. Fähigkeit des Menschen zu sprechen; das Sprechen als Anlage, als Möglichkeit des Menschen sich auszusprechen [...]; 2. das Sprechen, Rede [...]; 3. a) Art des Sprechens, Stimme, Redeweise [...], b) Ausdrucksweise, Stil [...]; 4. (historisch entstandenes und sich entwickelndes) System von Zeichen und Regeln, das einer Sprachgemeinschaft als Verständigungsmittel dient [...] (Duden. Deutsches Universalwörterbuch 2011: 1650).

- Deutsch: Zunge - 1. dem Schmecken und der Hervorbringung von Lauten (beim Menschen bes. dem Sprechen) dienendes und an der Nahrungsaufnahme, am Kauen und am Schlucken beteiligtes, am Boden der Mundhöhle befindliches, oft sehr bewegliches, mit Schleimhaut bedecktes muskulöses Organ der meisten Wirbeltiere und des Menschen [...]; 8. etwas, was in seiner Form an eine Zunge erinnert [...]; 10. Sprache [...] (Duden. Deutsches Universalwörterbuch 2011: 2077).

Es gibt aber spezifische Kontexte, in welchen das uneigentliche Wort Zunge das eigentliche Wort Sprache ersetzen kann. Es handelt sich um eine Metapher (z.B. Sie hat eine spitze, scharfe, lose, böse Zunge. - Sie neigt zu spitzen, scharfen usw. Äußerungen, Bemerkungen; Er hat eine falsche Zunge. - Er ist ein Lügner; Sie spricht mit doppelter Zunge. - Sie ist unaufrichtig, jemandem die Zunge lösen - jemanden zum Sprechen bringen; So weit die deutsche Zunge klingt - gehoben überall, wo man Deutsch spricht; alle Länder spanischer Zunge - poetisch Sprache, in fremder Zunge - poetisch Sprache (vgl. Wörter und Wendungen. Wörterbuch zum deutschen Sprachgebrauch 1990: 806; Duden. Deutsches Universalwörterbuch 2011: 2077), die deshalb möglich ist, weil im 
Deutschen das Hauptsprechorgan und die Sprache selbst durch zwei verschiedene Lexeme ausgedrückt werden. Im Polnischen dagegen wird beides durch dasselbe Lexem język ausgedrückt.

Eine andere Sprache differenziert häufig in ihrem Lexikon stärker als die Muttersprache des Lernenden und verfügt über zwei oder mehr Wörter, wo die Muttersprache des Lernenden nur eines hat. Bei der Übersetzung muttersprachiger Wörter und Wortgruppen in die Fremdsprache ergibt sich oft das Problem, das einer Ausgangssprachen-Einheit mehrere Zielsprachen-Einheiten gegenüberstehen (Leisi 1985: 41f.).

Jeder Kode, damit auch der Sprachkode, funktioniert als Zeichensystem auf der Grundlage von Vergleichs- und Bezugsgrößen. Immer sind die kommunikativen Bedürfnisse der Sprachgemeinschaft ein wesentliches Kriterium für die auBersprachlichen Bezüge der Sprachgemeinschaft.

Die Sprachen spiegeln denn nicht die Wirklichkeit, sondern es handelt sich um spezifisch menschliche, auswählende, begrifflich bündelnde und wertende Gliederungen, mit denen die Sprachgemeinschaften ihren jeweiligen Bedürfnissen und ihrem kulturbedingten theoretischen und praktischen Erkenntnisinteresse zu entsprechen suchen (vgl. Gipper 1993: 130).

Zusammenfassend lässt sich Folgendes andeuten: Wir haben es hier mit dem Interferenzfehler zu tun, der darauf zurückzuführen ist, dass die Deutsch lernenden Polen sich dem Einfluss ihrer Muttersprache nicht entziehen können, auch wenn sie fortgeschritten sind. Aus dem angeführten lexikalischen Fehler ergibt sich, dass die meisten Lernenden in ihrer Muttersprache denken und auf muttersprachliche Strukturen zurückgreifen, indem sie das deutsche Äquivalent Wort für Wort übersetzen. Wenn sie dabei kulturelle Besonderheiten in der gelernten Fremdsprache nicht kennen, begehen sie Fehler. In diesem Zusammenhang ist zu bemerken, dass das Übersetzen nicht die Transkodierung von Wortbedeutungen aus einer Sprache in eine andere ist, sondern eine komplexe Handlung, während der man die kulturellen und landesspezifischen Unterschiede, Lebenserfahrungen, eigenkulturell geprägte Konzepte von der Welt und die Weltbewertung berücksichtigen soll. Dabei ist ein tieferes Verstehen des Eigenen und des Fremden wichtig, weil sich in Wortbedeutungen kulturspezifische Erfahrungen und Wertvorstellungen niederschlagen. Wie oben ausgeführt wurde, ist die für jede Sprachgemeinschaft typische Begriffsbildung der Umwelt von Sprache zu Sprache unterschiedlich sprachlich repräsentiert, d.h. die sprachliche Repräsentation spiegelt eine von Sprache zu Sprache unterschiedliche Welterfassung wider, indem sie andere Merkmale der jeweiligen Sprache wiedergibt. Zwischen Sprachen gibt es kulturelle und landesspezifische Unterschiede. Die eine Sprache gibt die Wirklichkeit anders als die andere wieder. Es gibt also kein Kriterium, nach dem man sagen kann, dass die deutsche Sprache Unrecht hat, wenn sie Zunge und Sprache unterscheidet, während zum Beispiel das Polnische beide deutschen Lexeme unter język zusammenfasst. 


\section{Bibliographie}

Anusiewicz, Janusz: Problematyka językowego obrazu świata w pogladach niektórych językoznawców i filozofów niemieckich XX wieku. In: Bartmiński, Jerzy (Hrsg.): Językowy obraz świata. Lublin 1999. S. 261-289.

Bawej, Izabela: Muttersprachliche Interferenz im Lichte der kontrastiven Fehleranalyse am Beispiel Polnisch-Deutsch. Bydgoszcz 2014.

Blank, Andreas: Einführung in die lexikalische Semantik für Romanisten. Tübingen 2001.

Brunzel, Peggy: Kulturbezogenes Lernen und Interkulturalität. Zur Entwicklung kultureller Konnotationen im Französischunterricht der Sekundarstufe I. Tübingen 2002.

Dederichs, Teresa: Weltansicht. Ein semantisch-konnotativer Vergleich zwischen westdeutschen und rußlanddeutschen Jugendlichen. München/Berlin 1997.

Diller, Hans Jürgen / Kornelius, Joachim: Linguistische Probleme der Übersetzung. Tübingen 1978.

Duden. Deutsches Universalwörterbuch. Mannheim 2011.

Gipper, Helmut: Wilhelm von Humboldts Bedeutung für Theorie und Praxis moderner Sprachforschung. Münster 1992.

Gipper, Helmut: Eigen- und Stellenwert der Wortinhalte in Feld und Wortschatz. Münster 1993.

Grzegorczykowa, Renata: Pojęcie językowego obrazu świata. In: Bartmiński, Jerzy (Hrsg.): Językowy obraz świata. Lublin 1999. S. 39-46.

Humboldt, Wilhelm von: Wilhelm von Humboldts Werke. In: Leitzmann, Albert (Hrsg.). Bd. 1-17. Berlin: Behr's Verlag. Photomechanischer Nachdruck 1968.

Langenscheidts Taschenwörterbuch der polnischen und der deutschen Sprache. Berlin und München 1994.

Leisi, Ernst: Praxis der englischen Semantik. Heidelberg 1985.

Löbner, Sebastian: Semantik. Eine Einführung. Berlin 2003.

Lyons, John: Die Sprache. München 1983.

Lyons, John: Einführung in die moderne Linguistik. München 1989.

Nowy słownik języka polskiego. (Hrsg.) Sobol, Elżbieta. Warszawa 2002.

Schwarz, Monika / Chur, Jeanette: Semantik. Ein Arbeitsbuch. Tübingen 1983.

Spillmann, Hans Otto: Einführung in die germanistische Linguistik. Berlin 2000.

Tabakowska, Elżbieta: Bariery kulturowe sa zbudowane z gramatyki. In: Lewicki, Roman (Hrsg.): Przekład - język - kultura. Lublin 2002. S. 25-34.

Trier, Jost: Das sprachliche Feld. In: Antal, Laszlo (Hrsg.): Aspekte der Semantik. Zu ihrer Theorie und Geschichte 1662-1970. Frankfurt am Main 1972. S. 79-114.

Weiss, Daniel Mark: Beeinflussung der Wahrnehmung durch Zweisprachigkeit. Frankfurt am Main 1996.

Wierzbicka, Anna: Stowa klucze. Różne języki - różne kultury. Przekład Izabela Duraj-Nowosielska. Warszawa 2007.

Wörter und Wendungen. Wörterbuch zum deutschen Sprachgebrauch. Leipzig 1990.

\section{Abstracts}

Im Vordergrund folgender Erörterungen steht die Frage, wie sich Sprachen in semantischer Hinsicht auf kulturabhängige Art und Weise unterscheiden und ob das Deutsche Recht hat, wenn es Sprache und Zunge unterscheidet. Deswegen wird beispielhaft aufgezeigt, dass in der einen Sprache Bedeutungsabgrenzungen vorkommen, die es in einer anderen nicht gibt. Dann wird zur Sprache gebracht, in welchen Kontexten die Wörter Sprache und Zunge gebraucht werden können. Auf dieser Grund- 
lage wird dargestellt, dass in jeder Sprache und in jeder Kultur eine ganz bestimmte Einteilung und Verfügbarmachung der Wirklichkeit zum Ausdruck kommt. Jede Kultur entwickelt sich in die Richtung, die ihr von ihren jeweiligen Umweltbedingungen vorgegeben wird.

Schlüsselwörter: Kultur, Weltinterpretation, sprachliche Unterschiede

\title{
Is the distinction between Sprache and Zunge in German justified? Some information about the cultural and lexical differences in the image of extra-lingual reality
}

The present paper discusses the issue of culture-specific semantic differences between languages, as well as the question whether the German language has the right to differentiate between Sprache and Zunge. Using exemplary material, it is shown that semantic boundaries of one language are absent from another. Next the contexts in which the words Sprache and Zunge are used are discussed. On this basis it is explained that each language and each culture offers a specific way of classifying reality and making it available. Each culture develops in the direction which is dictated by specific environmental conditions.

Keywords: culture, image of the world, lexical differences

\author{
Izabela Bawej \\ Uniwersytet Kazimierza Wielkiego w Bydgoszczy \\ Instytut Neofilologii i Lingwistyki Stosowanej \\ ul. Grabowa 2 \\ 85-601 Bydgoszcz \\ Polen \\ E-Mail: iza_bawej@poczta.onet.pl
}

